\title{
Nuclear physics insights for new-physics searches using nuclei: Neutrinoless $\beta \beta$ decay and dark matter direct detection
}

\author{
Javier Menéndez $z^{1, a}$ \\ ${ }^{1}$ Department of Physics, The University of Tokyo, Hongo, Bunkyo-ku, Tokyo 113-0033, Japan
}

\begin{abstract}
Experiments using nuclei to probe new physics beyond the Standard Model, such as neutrinoless $\beta \beta$ decay searches testing whether neutrinos are their own antiparticle, and direct detection experiments aiming to identify the nature of dark matter, require accurate nuclear physics input for optimizing their discovery potential and for a correct interpretation of their results. This demands a detailed knowledge of the nuclear structure relevant for these processes. For instance, neutrinoless $\beta \beta$ decay nuclear matrix elements are very sensitive to the nuclear correlations in the initial and final nuclei, and the spin-dependent nuclear structure factors of dark matter scattering depend on the subtle distribution of the nuclear spin among all nucleons. In addition, nucleons are composite and strongly interacting, which implies that many-nucleon processes are necessary for a correct description of nuclei and their interactions. It is thus crucial that theoretical studies and experimental analyses consider $\beta$ decays and dark matter interactions with a coupling to two nucleons, called two-nucleon currents.
\end{abstract}

\section{Introduction}

Neutrinos and dark matter are two of the most promising candidates for new physics beyond the Standard Model of particle physics. Because they are both charge neutral and massive, it is possible that neutrinos and antineutrinos would be the same particle, in which case neutrinos would be labeled as Majorana particles [1]. This property — very hard to test because neutrinos are so light— would imply the violation of lepton number, a relation which goes in both directions: the violation of lepton number would establish neutrinos to be Majorana particles. In turn, this may have important consequences for the understanding of the baryonic matter-antimatter asymmetry observed in the universe, as in most models the difference between baryon and lepton number is conserved.

Unveiling the origin of dark matter stands as one of the biggest challenges in physics. The existence of dark matter has been certified by very different astrophysical observations - galactic rotation velocities, gravitational lensing, anysotropies of the cosmic microwave background - but the nature of dark matter is still unknown [2]. Observations have constrained some of its properties, such that it must be neutral to the electromagnetic interaction - to a very good approximation at least - that it should be cold or warm to allow for galaxy structure formation, and that it amounts to more than $80 \%$ of the mass content of the universe, and roughly a quarter of the energy content.

a e-mail: menendez@nt.phys.s.u-tokyo.ac.jp 
Ideally one would like to answer these questions on the nature of neutrinos and dark matter in the laboratory. Experimental programs searching for neutrinoless $\beta \beta$ decay - the lepton number violating process most likely to be observed - and the direct detection of dark matter are being pursued vigorously, and impressive advances are permanently being reported, with present experimental sensitivities reaching half-lives longer than $T_{1 / 2}^{0 \nu \beta \beta}=10^{26}$ years [3] for neutrinoless $\beta \beta$ decay, and excluding scattering cross-sections off nuclei smaller than $\sigma_{\chi \mathcal{N}}=10^{-40} \mathrm{~cm}^{2}$ [4] for searches of dark matter. Further improvements are expected in the near future, as next generation experiments are planned to use over a tonne of source or target material with increasingly reduced backgrounds.

Neutrinoless $\beta \beta$ decay and dark matter direct detection experiments have in common that they are looking for the decay and the scattering off atomic nuclei, respectively. Therefore, the design - for example, the choice of source or target material - and the interpretation of the experimental results in principle depends on the nuclear physics of the process at study. In the case of neutrinoless $\beta \beta$ decay, the value of the nuclear matrix element driving the transition relies on the accurate nuclear structure description of the initial and final nuclei, and on the weak-interaction diagrams considered at the nucleon level [5]. In the case of dark matter detection, a correct interpretation of the experimental results taking into account all relevant nuclear structure factors depends on considering all possible interactions of nuclei with dark matter particles. In particular, $\beta$ decays and dark matter interactions with a coupling to two nucleons, in addition to the leading contributions which only involve a single nucleon, can be significant.

\section{Neutrinoless $\beta \beta$ decay}

\section{$2.1 \beta \beta$ decay: two-neutrino and neutrinoless cases}

The existence of $\beta \beta$ decay is a consequence of the nuclear pairing interaction, which makes nuclei with an even number of protons, or an even number of neutrons, more bound than nuclei with one or two - a proton and a neutron- unpaired nucleons. As a result, in some cases it is energetically favorable for a nucleus to decay along a given isobaric chain — set of nuclei with the same number of nucleons - via a second-order $\beta \beta$ decay, instead of the usual single- $\beta$ decay channel. For the case of ${ }^{76} \mathrm{Ge}$ the decay scheme is shown in figure $1 . \beta \beta$ decay with the emission of two antineutrinos besides two electrons, a lepton-conserving process permitted by the weak interaction, has been observed in

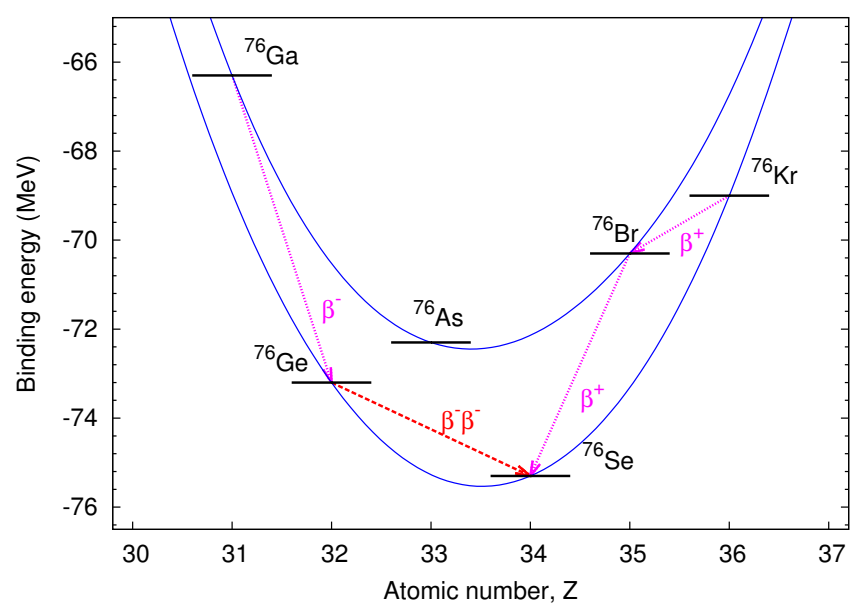

Figure 1. Nuclear binding energies and decay scheme of the nucleon number $A=76$ isobars, as a function of the atomic number $Z$. In a typical situation, isotopes decay to the lowest-energy nucleus via single $\beta^{-}$or $\beta^{+}$decays. For the special case of ${ }^{76} \mathrm{Ge}$, however, single- $\beta$ decay is energetically forbidden, leaving $\beta \beta$ decay as the only channel available to reach the stable nucleus ${ }^{76} \mathrm{Se}$. 
a dozen of cases, favored by a larger energy difference between the initial and final nuclei. The measured half-lives are of the order of $T_{1 / 2}^{2 v \beta \beta} \sim 10^{19}-10^{21}$ years [6].

Neutrinoless $\beta \beta$ decay does not involve the emission of neutrinos, and it therefore violates lepton number. It demands neutrinos to be Majorana particles. The neutrinoless case is at least five or six orders of magnitude slower than the two-neutrino $\beta \beta$ decay permitted by the Standard Model. In the standard scenario where the decay is mediated by the exchange of the three known light neutrinos this is because the decay rate is proportional to the neutrinos masses, which are tiny compared to those of any other lepton. In other scenarios involving new physics, the reason is the large mass of the exchange particles, or the small coupling of the new physics with the Standard Model sector. In the standard case the neutrinoless $\beta \beta$ decay half-life can be written as [1]

$$
\left[T_{1 / 2}^{0 \nu \beta \beta}\right]^{-1}=G^{0 \nu \beta \beta}\left|M^{0 \nu \beta \beta}\right|^{2} m_{\beta \beta}^{2},
$$

which naturally includes a phase space factor $G^{0 v \beta \beta}$ that takes into account the kinematics, a nuclear matrix element $M^{0 v \beta \beta}$ that contains the relevant nuclear physics of the decay, and a third part $m_{\beta \beta}=\sum_{k} m_{k} U_{e k}^{2}$ that encodes the new-physics scale - the neutrino masses $m_{k}$ - and also includes the mixing of electron neutrinos with other flavors, $U_{e k}$. The nuclear matrix element can be decomposed according to the spin structure of the operator [1]:

$$
M^{0 v \beta \beta}=M^{G T}-\frac{g_{V}}{g_{A}} M^{F}+M^{T}
$$

where the dominant term is the so-called Gamow-Teller component, $M^{G T}$.

\subsection{Nuclear matrix elements: nuclear structure}

Neutrinoless $\beta \beta$ decay nuclear matrix elements $M^{0 v \beta \beta}$ have to be obtained by nuclear structure calculations evaluating the transition operator between the initial and final nuclear states. The present status of these calculations is illustrated in figure 2. Unfortunately different nuclear structure approaches disagree in their predicted matrix elements for every $\beta \beta$ decay candidate by up to a factor three. This is a clear evidence that the unavoidable approximations present in solving the nuclear many-body problem are not under control when studying neutrinoless $\beta \beta$ decay [5]. In contrast, it should be noted that the same different many-body methods in general agree when studying other nuclear structure properties such as excitation spectra or electromagnetic transitions.

It is thus critical to clarify the actual value of neutrinoless $\beta \beta$ decay nuclear matrix elements. A first avenue for doing so is to test the calculations by finding correlations of matrix elements with other measured quantities. Despite efforts in this direction [18], no single observable has been found to be especially correlated to neutrinoless $\beta \beta$ decay. A promising process is two-neutrino $\beta \beta$ decay, which shares with the neutrinoless case the initial and final states and for which there is experimental data. It must be taken into account, however, that the relevant momentum transfers are very different in the two-neutrino and neutrinoless decays, $q \sim 1 \mathrm{MeV}$ for the former and $q \sim 100 \mathrm{MeV}$ for the latter. Unfortunately most many-body approaches cannot predict two-neutrino $\beta \beta$ decay, because an accurate calculation involves dealing with the intermediate odd-proton-odd-neutron system - for instance, the nucleus ${ }^{76} \mathrm{Sb}$ in figure $1-$ and this is a more involved nuclear structure calculation than the one needed for even-even nuclei. Other approaches like quasiparticle random phase approximation method use the two-neutrino $\beta \beta$ decay half-life to fix a free parameter in their model and are not predictive for this decay. The only remaining many-body approach is the shell model, which actually predicted the two-neutrino $\beta \beta$ decay rate of ${ }^{48} \mathrm{Ca}$ [19] in good agreement with the subsequent measurement a few years later [20]. However, the accepted experimental value has been challenged by a 


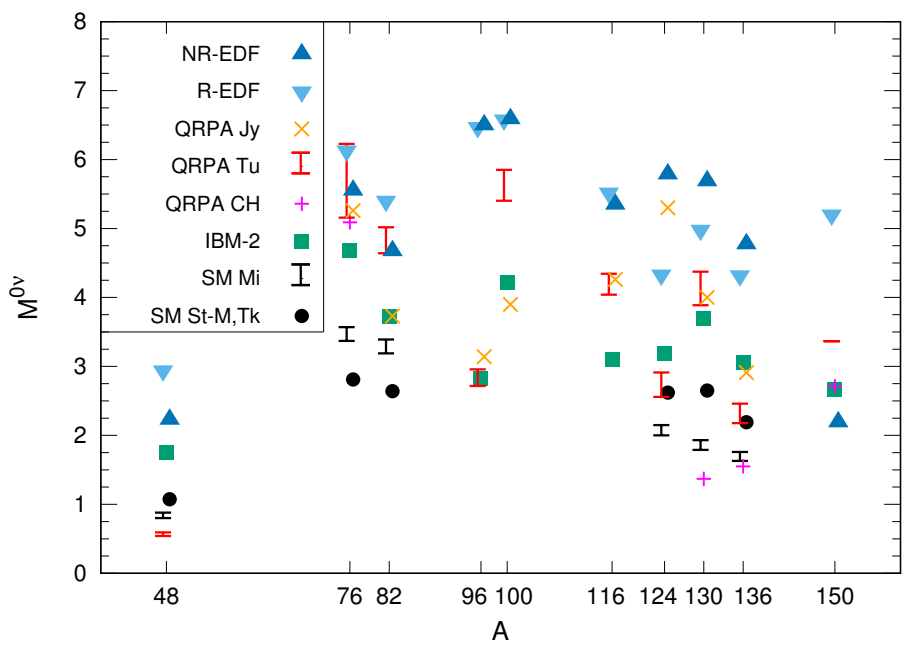

Figure 2. Nuclear matrix elements $M^{0 v \beta \beta}$ for neutrinoless $\beta \beta$ decay candidates, shown as a function of the nucleon number $A$, calculated with different nuclear structure methods. Blue symbols indicate energy density functional (EDF) calculations, nonrelativistic [7] (triangles) and relativistic [8,9] (down triangles). Orange crosses, red bars and magenta plus signs stand for quasiparticle random phase approximation (QRPA) results obtained by the Jyväskylä [10], Tübingen [11, 12] and Chapel Hill [13] groups, respectively. Green squares show interacting boson model (IBM) values [14]. Finally, black bars and circles represent the shell model (SM) results obtained by the Michigan [15] and Strasbourg-Madrid [16] groups, respectively, except for the ${ }^{48} \mathrm{Ca}$ black circle which shows the recent shell model calculation by the Tokyo group [17].

recent measurement [21], which would bring the shell model prediction into an overestimation of the corresponding matrix element. The description of the two-neutrino $\beta \beta$ decay rate of ${ }^{136} \mathrm{Xe}$ within the shell model is also under discussion [22, 23].

In recent years an effort has been made to understand the origin of the differences between manybody approaches by comparing the systematic calculations of matrix elements, even if the associated $\beta \beta$ decays make little or no sense experimentally. For instance a comparison between shell model and energy density functional matrix elements which in general disagree the most between different methods, as shown in figure 2, but restricting the calculations to uncorrelated states - fully composed of proton-proton and neutron-neutron angular momentum $J=0$ pairs in the shell model, and limited to spherical states in the energy density functional calculation - showed that the uncorrelated nuclear matrix element disagreement was limited to $30 \%$ or less [25]. This is illustrated by figure 3 and is a very significant improvement over the factor three disagreement in figure 2 . The matrix element values are fixed by the strength of the - neutron-neutron and proton-proton- pairing interaction.

This finding suggests that it is the different way in which the many-body approaches include nuclear structure correlations which is behind much of the disagreement in neutrinoless $\beta \beta$ decay nuclear matrix elements. Proton-neutron pairing has been known to be important for $\beta$ and $\beta \beta$ decay decays for a long time [24]. Recent shell model calculations based on a separable effective interaction have confirmed this extreme, showing that nuclear matrix elements are overestimated if proton-neutron pairing —-more precisely, isoscalar pairing — is excluded [26]. Since at the moment isoscalar pairing is not fully captured by energy density functional and interacting boson model calculations, this may 


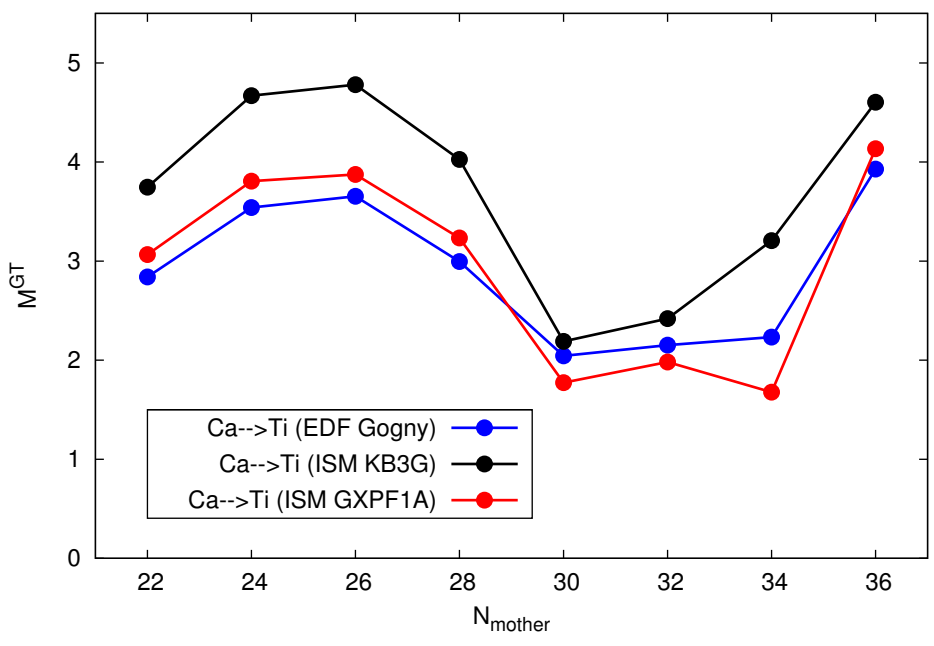

Figure 3. Gamow-Teller component of the neutrinoless $\beta \beta$ decay matrix element, $M^{G T}$, for the decay of calcium into titanium isotopes, as a function of the neutron number of the calciums $N_{\text {mother }}[25]$. Results from an energy density functional calculation (blue) are compared to shell model results obtained with two different effective interactions (black and red).

be a reason for the discrepancies shown in figure 2. Dedicated studies incorporating these correlations in the corresponding nuclear matrix element calculations are needed.

Another cause of missing correlations is the limitation of the configuration space, this is, the single-particle orbitals that nucleons are permitted to occupy. For instance, the shell model and the interacting boson model only solve explicitly the nuclear many-body problem in a limited configuration space around the Fermi surface, and include the effect of the remaining configurations approximately. In order to quantify the effect of the missing correlations, a many-body perturbation theory estimate found relatively moderate increases of less than $50 \%$ for the lightest $\beta \beta$ decay emitters ${ }^{48} \mathrm{Ca},{ }^{76} \mathrm{Ge}$ and ${ }^{82} \mathrm{Se}$, for which the estimation is easier [27, 28].

A more rigorous estimation is the recent computation of the $\beta \beta$ decay of ${ }^{48} \mathrm{Ca}$ extending the shell model configuration space from one to two major harmonic oscillator shells - limited to $2 \hbar \omega$ excitations - reducing the core of the shell model calculation from ${ }^{40} \mathrm{Ca}$ to ${ }^{16} \mathrm{O}$. By doing so, many previously excluded configurations were permitted, and the dimension of the problem increases from less than $10^{6}$ to over $10^{9}$. The impact on the nuclear matrix element is, however, moderate, with a $30 \%$ enhancement due to previously-missing cross-shell pairing correlations [17]. Interestingly, a cancellation occurs between the general enhancement produced by additional pairing correlations and the contribution of particle-hole excitations. A similar cancellation is expected to be at play for other $\beta \beta$ decay candidates as well. Nevertheless, explicit calculations are needed. Shell model calculations in extended configuration spaces can benefit from using the Monte Carlo shell model technique, which recently has studied configuration spaces with dimension over $10^{23}$ [29].

Finally, other many-body approaches than those represented in figure 2 can shed light into $\beta \beta$ decay nuclear matrix elements. In particular, in the last decade nuclear ab initio calculations - those solving the many-body problem for all nucleons in the system, with nuclear forces fitted only to light nuclei- have been able to perform calculations up to medium-mass isotopes, in many cases achieving very good agreement to experiment [30]. Even before ab initio calculations are available for $\beta \beta$ decay emitters, these many-body techniques can be used to benchmark $\beta \beta$ decay matrix elements in lighter or less-correlated systems to gain insight on the relevant physics for this process. Not only ab initio calculations are more controlled than the phenomenological ones available so far for $\beta \beta$ decay, but they also in principle allow for the estimation of theoretical nuclear matrix element uncertainties, a very valuable information for the interpretation of the experimental results [5]. 


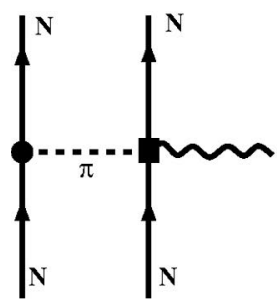

a)

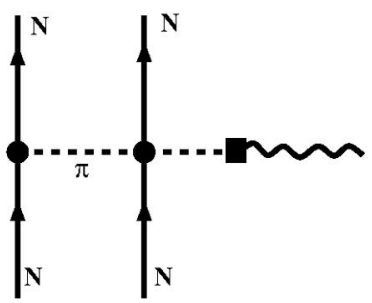

b)

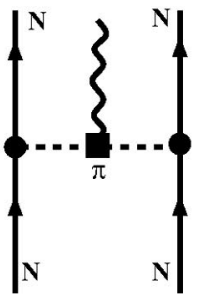

c)

Figure 4. Leading long-range two-body currents contributing to neutrinoless $\beta \beta$ decay [32-34] and dark matter scattering off nuclei [35-38]. Solid and dashed lines indicate nucleons $(N)$ and pions $(\pi)$, respectively, and wavy lines stand for the external source, in this work weak and dark matter interactions that can be approximated as contact couplings. Diagrams a) and b) stand for one-pion exchange and one-pion exchange pion-pole couplings appearing in the leading axial two-body current. Diagram c) represents the pion-in-flight coupling in the leading vector and scalar two-body currents. All three diagrams enter in both processes.

\subsection{Two-body currents}

The nuclear matrix discussed so far are based on the standard one-body operator: each weak interaction vertex involves only one nucleon. However, because nucleons are composite particles that strongly interact to each other, the single- $\beta$ decay hadronic current takes the general form [31]

$$
\mathbf{J}=\sum_{i=1} \mathbf{J}_{i, 1 \mathrm{~b}}+\sum_{i<j} \mathbf{J}_{i j, 2 \mathrm{~b}}+\cdots
$$

Diagrams involving two nucleons, represented in figure 4, are a correction to the leading one-body terms. Two-body corrections have been intensively explored in nuclei lighter than those undergoing $\beta \beta$ decay, where they have been found to be relevant for electromagnetic [39] and weak [40] transitions.

Two-body weak currents have been mostly studied in the context of small momentum transfers, such as single- $\beta$ decay. Since calculations are quite demanding, most works have been limited to relatively light nuclei, up to about oxygen, or relatively rough approximations, such as the normalordering of the two-body currents with respect to an isospin-symmetric Fermi gas. These studies indicate that two-body currents tend to cancel the contribution of the leading one-body operator, which means that they are a contribution to the so-called " $g_{A}$ quenching". The " $g_{A}$ quenching" stands for the empirical fact that nuclear many-body calculations need to reduce the strength of the spin-isospin operator in order to agree with the experimental half-lives of Gamow-Teller $\beta$ transitions. However, the size of the two-body contributions is unclear, with results ranging for less than $10 \%$ for carbon and oxygen isotopes [41], to about $30 \%$ in larger systems but with a more rough normal ordering [32]. Especially important are the uncertainties in the short-range two-body currents - not shown in figure 4 . The " $g_{A}$ quenching" should be carefully studied in lighter systems where dedicated ab initio calculations with different approaches are feasible.

In contrast to single- $\beta$ decay, where the relevant momentum transfer is $q \sim 1 \mathrm{MeV}$, in neutrinoless $\beta \beta$ decay transferred momenta can reach $q \sim 100 \mathrm{MeV}$, because of the virtual nature of the exchanged neutrinos. This different momentum-transfer regime can have important consequences in the effect of two-body currents, because several pion-exchange (figure 4 a) and pion-pole (figure 4 b) contributions contribute at finite $q$ [33]. Present results show that $q$-dependent two-body contributions partially cancel other two-body terms $[32,34,42]$, resulting in a smaller reduction of Gamow-Teller matrix elements in neutrinoless $\beta \beta$ decay than in single $\beta$ and two-neutrino $\beta \beta$ decays. 


\section{Dark matter scattering off nuclei}

\subsection{Dark matter-nucleon interactions and scattering cross-section}

Direct detection dark matter searches are motivated by weakly interacting massive particles (WIMPs), promising dark matter constituents that are predicted to naturally account for the observed dark matter density. The expected WIMP masses are $M_{\chi} \sim 1-1000 \mathrm{GeV}$, the mass scale of nuclei. Therefore experiments sensitive to these dark matter masses use nuclei as target. Similarly lighter masses $M_{\chi} \sim$ $1 \mathrm{MeV}$ are probed via the scattering of dark matter off electrons [2].

WIMPs can interact with nuclei in many ways. However, commonly the interactions suppressed by the small WIMP velocities $-v / c \sim 10^{-3}$ - or the momentum transfer of the scattering -much smaller than $M_{\chi}$ and the nucleon mass - are not considered. This leads to two possibilities [43]: the direct coupling of the dark matter and nuclear densities, called spin-independent scattering, and the coupling of the dark matter and nuclear spins, referred to as spin-dependent scattering. Spinindependent scattering is favored from the nuclear physics side because it is coherent: at vanishing momentum transfer it receives contributions from all nucleons in the nucleus. In contrast, in spindependent responses, on average only the spin of one nucleon contributes, because the spins of two nucleons tend to couple to a spin-zero pair due to the pairing interaction. Other things being equal, coherent scattering is expected to be enhanced by a factor $A^{2}$ with respect to the spin-dependent case.

A more complete description of the possible WIMP interactions with nuclei has been worked out in a nonrelativistic effective field theory (EFT) [44, 45]. By constructing all possible interactions that can be built from the WIMP and nucleon spins, the momentum transfer and the WIMPs relative velocity, a set of independent operators $O_{i}$ is derived at the nucleon level. At the nuclear level, however, not all operators $O_{i}$ leave distinct signatures, because there are only six independent nuclear responses, which may interfere between them. In addition, the interactions of dark matter with two nucleons need to be considered [35-37], see figure 4. Two-body currents can contribute significantly to dark matter scattering, both for a coherent response, and for spin-dependent interactions.

In general, the WIMP-nucleus cross-section can be written as [38]

$$
\frac{\mathrm{d} \sigma_{\chi \mathcal{N}}}{\mathrm{d} \mathbf{q}^{2}} \propto\left|\sum_{i} c_{i} \zeta_{i} \mathcal{F}_{i}\right|^{2}+\left|\sum_{i} \hat{c}_{i} \hat{\zeta}_{i} \hat{\mathcal{F}}_{i}\right|^{2}+\cdots
$$

Here $\zeta, \hat{\zeta}$ are kinematic factors, $c, \hat{c}$ encode the hadronic physics and particle physics - for instance the Wilson coefficients coupling WIMPs with quarks and gluons- and $\mathcal{F}, \hat{\mathcal{F}}$ represent the nuclear physics: its square gives the nuclear structure factors. As shown in Eq. (4), contributions may interfere or not. In particular, the usual spin-independent and spin-dependent terms do not interfere.

\subsection{Coherent (spin-independent) scattering}

Spin-independent — coherent - scattering can be generalized by considering all one-nucleon operators proposed by the nonrelativistic EFT. The most relevant terms are characterized by an enhancement of the cross-section, driven by the nuclear physics, and reflected in the structure factors $\mathcal{F}^{2}, \hat{\mathcal{F}}^{2}$.

In the nonrelativistic EFT there are two nuclear responses which can be coherent [44, 45]. First, the standard spin-independent response, denoted as $M$, corresponding to the operator $O_{1}$ and other subleading operators. Second, the nuclear response associated with the operator $O_{3}$, denoted by $\Phi^{\prime \prime}$, which is partially coherent. In this case, all nucleons with spin aligned with the angular momentum $l$ contribute coherently. Nucleons with spin antiparallel to $l$ cancel this contribution, but single-particle states with parallel spin are lowered in energy due to the nuclear spin-orbit force, leaving part of the 


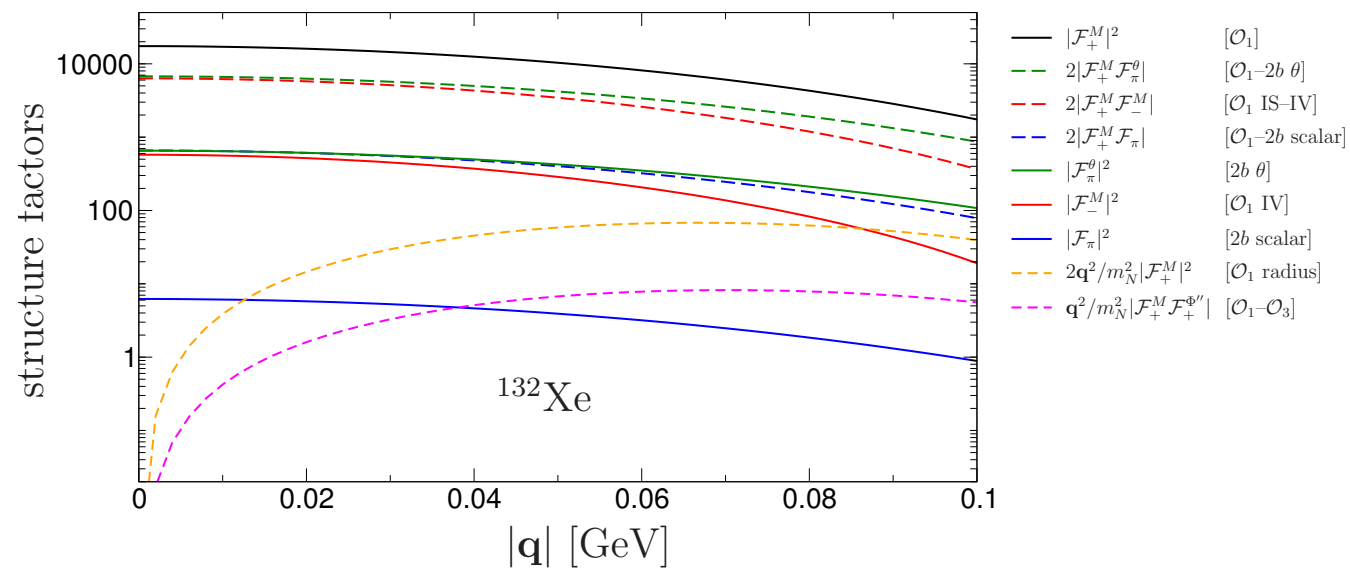

Figure 5. Structure factors associated with different WIMP interactions with ${ }^{132} \mathrm{Xe}$, as a function of the momentum transfer $q$ [38]. The solid (red) black and line shows the isoscalar (isovector) $O_{1}$ contributions. The green and blue solid lines denote the two-body current contributions with scalar and $\theta$-term couplings, respectively, while the dashed lines with the same colors stand for their interference with the isoscalar $O_{1}$ term. Orange (magenta) dashed lines denote the interference of the one-nucleon radius corrections $\left(O_{3}\right.$ operator) with the $O_{1}$ term.

antiparallel-spin states empty and preventing a complete cancellation. Since this coherent term is spin dependent, the standard terminology must be generalized: it is more appropriate to speak of coherent scattering instead of spin-independent scattering.

Besides the nuclear structure aspects - coherence - the hadronic physics is also crucial to set the hierarchy among different terms. Chiral EFT [46, 47], an effective theory of the underlying interaction that binds nucleons, quantum chromodynamics (QCD), is valid at the energy and momentum scales of WIMP scattering and incorporates the physics of the chiral symmetry of QCD. Chiral EFT also describes consistently the interactions with external probes [39]. By formulating the WIMP-nucleon interactions in the chiral EFT framework, including scalar, pseudoscalar, vector and axial contributions in the WIMP and hadronic sectors, the leading operators are predicted and can be matched into the nonrelativistic EFT basis [33]. In addition, chiral EFT predicts the consistent interactions of WIMPs with two nucleons - two-body currents. The relative importance of the different contributions can be studied by calculating the corresponding structure factors for the one- and two-body operators, assuming similar contributions from the particle physics Wilson coefficients.

Figure 5 shows the structure factors for the most important one- and two-nucleon contributions to the coherent WIMP scattering off ${ }^{132} \mathrm{Xe}$. This is the most abundant isotope in xenon, the target used in experiments giving the present best limits on WIMP-nucleus scattering. Solid lines in figure 5 represent the most important individual contributions, which are the isoscalar $O_{1}$ term -routinely used in experimental analyses - its isovector counterpart - with opposite coupling to protons and neutrons, usually included to account for apparently conflicting results in experiments using different isotopes - and two different couplings of the WIMP to two nucleons: through a scalar coupling, and through a coupling to the trace anomaly of the energy-momentum tensor $-\theta$ term. As a result, the following extension of the cross-section for spin-independent scattering is proposed [38]

$$
\frac{\mathrm{d} \sigma_{\chi \mathcal{N}}^{\mathrm{SI}}}{\mathrm{d} \mathbf{q}^{2}}=\frac{1}{4 \pi \mathbf{v}^{2}}\left|c_{+}^{M} \mathcal{F}_{+}^{M}\left(\mathbf{q}^{2}\right)+c_{-}^{M} \mathcal{F}_{-}^{M}\left(\mathbf{q}^{2}\right)+c_{\pi} \mathcal{F}_{\pi}\left(\mathbf{q}^{2}\right)+c_{\pi}^{\theta} \mathcal{F}_{\pi}^{\theta}\left(\mathbf{q}^{2}\right)\right|^{2},
$$


with each $c$ coefficient sensitive to a different corner of the parameter space of new-physics models.

The dashed lines of figure 5 take into account the interferences with the dominant term. When these are included, two additional contributions appear: radius, or momentum-dependent, corrections to the leading operator - that nevertheless probe a different combination of new-physics parametersand the partially coherent operator $O_{3}$, which turns out to give the leading correction among all the one-nucleon operators proposed in the nonrelativistic EFT. A further generalization of the coherent cross-section is therefore suggested [38]:

$$
\begin{aligned}
\frac{\mathrm{d} \sigma_{\chi \mathcal{N}}^{\mathrm{SI}}}{\mathrm{d} \mathbf{q}^{2}}=\frac{1}{4 \pi \mathbf{v}^{2}} \mid & \left(c_{+}^{M}-\frac{\mathbf{q}^{2}}{m_{N}^{2}} \dot{c}_{+}^{M}\right) \mathcal{F}_{+}^{M}\left(\mathbf{q}^{2}\right)+c_{\pi} \mathcal{F}_{\pi}\left(\mathbf{q}^{2}\right)+c_{\pi}^{\theta} \mathcal{F}_{\pi}^{\theta}\left(\mathbf{q}^{2}\right) \\
& +\left(c_{-}^{M}-\frac{\mathbf{q}^{2}}{m_{N}^{2}} \dot{c}_{-}^{M}\right) \mathcal{F}_{-}^{M}\left(\mathbf{q}^{2}\right)+\left.\frac{\mathbf{q}^{2}}{2 m_{N}^{2}}\left[c_{+}^{\Phi^{\prime \prime}} \mathcal{F}_{+}^{\Phi^{\prime \prime}}\left(\mathbf{q}^{2}\right)+c_{-}^{\Phi^{\prime \prime}} \mathcal{F}_{-}^{\Phi^{\prime \prime}}\left(\mathbf{q}^{2}\right)\right]\right|^{2} .
\end{aligned}
$$

Note that not all terms are independent, as for instance for a Majorana (Dirac) spin 1/2 WIMP there are only 4 (7) independent Wilson coefficients, so that a correlated analysis of several experiments would be required. A more practical analysis with data from a single experiment and taking limits on one operator at a time — for instance on Eq. (5) — should take this carefully into account.

\subsection{Spin-dependent and inelastic scattering}

Coherent —-spin-independent- scattering is not very sensitive to the detailed nuclear structure of the target nuclei. This is because at vanishing momentum-transfer, the structure factor of the leading term is $\mathcal{F}_{+}^{M}=A^{2}$, simply counting the number of nucleons. The momentum-transfer dependence is given by the nuclear density [48]. In contrast, a careful nuclear structure calculation is needed for spindependent scattering, which is very sensitive to the nuclear spin distribution among all nucleons [49].

Only odd- $A$ nuclei are sensitive to spin-dependent interactions: stable even- $A$ nuclei have spin zero due to nuclear pairing. Therefore, for a given nucleus this interaction is mostly sensitive to the nucleon species with an odd number of components, either protons or neutrons. For odd- $A$ xenon isotopes ${ }^{129} \mathrm{Xe}$ and ${ }^{131} \mathrm{Xe}$, with atomic number $Z=54$, neutrons carry most of the spin and the so-called "structure factor for neutrons" is orders of magnitude larger than the "structure factor for protons". This separation is actually not general as it simply refers to different combinations of isoscalar — same for neutrons and protons - or isovector - opposite - couplings. When considering only one-nucleon operators, however, the separation is valid at vanishing momentum transfer [43]:

$$
\mathcal{F}_{1 \mathrm{~b}}^{\mathrm{SD}}(q=0) \propto\left|\left(a_{0}+a_{1}\right)\left\langle\mathbf{S}_{p}\right\rangle+\left(a_{0}-a_{1}\right)\left\langle\mathbf{S}_{n}\right\rangle\right|^{2},
$$

with $a_{0 / 1}$ the isoscalar/isovector couplings and $\left\langle\mathbf{S}_{p / n}\right\rangle$ the proton/nucleon spin expectation values.

Two-body currents prevent the validity of the separation. As illustrated in figure 4, two-nucleon interactions do not distinguish between neutrons and protons, and therefore it is not possible to disentangle proton and neutron contributions. The structure factor can be generalized as [37]

$$
\mathcal{F}_{1 \mathrm{~b}+2 \mathrm{~b}}^{\mathrm{SD}}(q=0) \propto\left|\left(a_{0}+a_{1}[1+\delta]\right)\left\langle\mathbf{S}_{p}\right\rangle+\left(a_{0}-a_{1}[1+\delta]\right)\left\langle\mathbf{S}_{n}\right\rangle\right|^{2},
$$

where $\delta \sim-0.2$ [49] encodes the two-nucleon contributions and can be calculated with chiral EFT. As a result, with two-body currents, the so-called "structure factor for protons" — defined by $a_{0}=-a_{1}$ in Eq. (8) - is also sensitive to neutrons, and increases by over an order of magnitude with respect to the one-nucleon case because for xenon isotopes $\left\langle\mathbf{S}_{n}\right\rangle \gg\left\langle\mathbf{S}_{n}\right\rangle$. This has important practical consequences, because it makes exclusion limits obtained in experiments using xenon — which is more sensitive to 
neutrons - competitive in "proton cross-sections" with searches using target nuclei with odd number of protons - thus more sensitive to protons - such as fluorine [50].

Once dark matter has been detected, it is left to address the nature of the dark matter-nucleus interaction. Spin-dependent scattering can be useful in this respect, because it could be observed in the elastic and inelastic channels. The experimental inelastic signature is distinct from the elastic one - the nucleus $\gamma$ decays to the ground state - and can be realized if the target nuclei has low-lying excited nuclear states, such as the $40 \mathrm{keV}$ and $80 \mathrm{keV}$ first excited states in ${ }^{129} \mathrm{Xe}$ and ${ }^{131} \mathrm{Xe}$ [51, 52]. For coherent scattering, the inelastic channel is always suppressed by a factor $A^{2}$ with respect to the elastic channel [48], making it presently undetectable in practice. Therefore, the observation of an inelastic signal would clearly point out to a spin-dependent interaction.

\section{Summary}

The most impressive experimental efforts are being made to unveil the nature of neutrinos and dark matter in low-energy experiments using nuclei as a source or target. To make the most of these searches, comparable theoretical efforts are needed to understand the nuclear physics driving these processes. Neutrinoless $\beta \beta$ decay nuclear matrix element calculations differ, but the most sensitive nuclear structure correlations for the decay have been identified, and calculations in larger configuration spaces are underway. The effect of the weak interaction involving two nucleons can also be significant, and explain part of the so-called " $g_{A}$ quenching". Ab initio calculations in lighter systems can be performed to fully understand this "quenching". Analyses of dark matter searches should consider all possible interactions of WIMPs with nuclei. In particular, the coupling to two nucleons can have significant impact in both coherent and spin-dependent scattering. The observation of inelastic scattering is a promising way to determine the nature of the dark matter interaction with nuclei.

\section{Acknowledgements}

I would like to thank my collaborators J. Engel, D. Gazit, N. Hinohara, M. Hoferichter, Y. Iwata, P. Klos, G. Martínez-Pinedo, T. Otsuka, A. Poves, T. R. Rodríguez, N. Shimizu, A. Schwenk, Y. Utsuno and L. Vietze for very enlightening discussions and for making use of our common work for these proceedings. This work has been supported by an International Research Fellowship the Japan Society for the Promotion of Science (JSPS) and JSPS Grant-in-Aid for Scientific Research No. 26.04323.

\section{References}

[1] F. T. Avignone III, S. R. Elliott, and J. Engel, Rev. Mod. Phys. 80, 481 (2008)

[2] L. Baudis, J. Phys. G 43, 044001 (2016)

[3] A. Gando et al. [KamLAND-Zen Collaboration], Phys. Rev. Lett. 117, 082503 (2016)

[4] D. S. Akerib et al. [LUX Collaboration], Phys. Rev. Lett. 116, 161301 (2016)

[5] J. Engel and J. Menéndez, arXiv:1610.06548

[6] A. Barabash, Nucl. Phys. A 935, 52 (2015)

[7] N. López Vaquero, T. R. Rodríguez and J. L. Egido, Phys. Rev. Lett. 111, 142501 (2013)

[8] J. M. Yao, L. S. Song, K. Hagino, P. Ring and J. Meng, Phys. Rev. C 91, 024316 (2015)

[9] J. M. Yao and J. Engel, Phys. Rev. C 94, 014306 (2016)

[10] J. Hyvärinen and J. Suhonen, Phys. Rev. C 91, 024613 (2015)

[11] F. Šimkovic, V. Rodin, A. Faessler and P. Vogel, Phys. Rev. C 87, 045501 (2013) 
[12] D. L. Fang, A. Faessler and F. Šimkovic, Phys. Rev. C 92, 044301 (2015)

[13] M. T. Mustonen and J. Engel, Phys. Rev. C 87, 064302 (2013)

[14] J. Barea, J. Kotila and F. Iachello, Phys. Rev. C 91, 034304 (2015)

[15] M. Horoi and A. Neacsu, Phys. Rev. C 93024308 (2016)

[16] J. Menéndez, A. Poves, E. Caurier and F. Nowacki, Nucl. Phys. A 818, 139 (2009)

[17] Y. Iwata, N. Shimizu, T. Otsuka, Y. Utsuno, J. Menéndez, M. Honma and T. Abe, Phys. Rev. Lett. 116, 112502 (2016)

[18] S. J. Freeman, and J. P. Schiffer, J. Phys. G: Nucl. Part. Phys. 39, 124004 (2012)

[19] E. Caurier, A. P. Zuker, and A. Poves, Phys. Lett. B 252, 13 (1990)

[20] A. Balysh et al., Phys. Rev. Lett. 77, 5186 (1996)

[21] R. Arnold et al. [NEMO-3 Collaboration], Phys. Rev. D 93, 112008 (2016)

[22] E. Caurier, F. Nowacki, and A. Poves, Phys. Lett. B 71162 (2012)

[23] M. Horoi and B.A. Brown, Phys. Rev. Lett. 110222502 (2013)

[24] P. Vogel and M. R. Zirnbauer, Phys. Rev. Lett. 57, 3148 (1986)

[25] J. Menéndez, T. R. Rodríguez, G. Martínez-Pinedo and A. Poves, Phys.Rev. C 90, 024311 (2014)

[26] J. Menéndez, N. Hinohara, J. Engel, G. Martínez-Pinedo and T. R. Rodríguez, Phys. Rev. C 93, 014305 (2016)

[27] J. D. Holt and J. Engel, Phys. Rev. C 87, 064315 (2013)

[28] A. A. Kwiatkowski et al., Phys. Rev. C 89, 045502 (2014)

[29] T. Togashi, Y. Tsunoda, T. Otsuka and N. Shimizu, Phys. Rev. Lett. 117172502 (2016)

[30] K. Hebeler, J. D. Holt, J. Menendez and A. Schwenk, Ann. Rev. Nucl. Part. Sci. 65, 457 (2015)

[31] T. S. Park et al., Phys. Rev. C 67, 055206 (2003)

[32] J. Menéndez, D. Gazit, and A. Schwenk, Phys. Rev. Lett. 107, 062501 (2011)

[33] M. Hoferichter, P. Klos and A. Schwenk, Phys. Lett. B 746, 410 (2015)

[34] J. Menéndez, arXiv:1605.05059

[35] G. Prézeau, A. Kurylov, M. Kamionkowski and P. Vogel, Phys. Rev. Lett. 91, 231301 (2003)

[36] V. Cirigliano, M. L. Graesser and G. Ovanesyan, JHEP 1210, 025 (2012)

[37] J. Menéndez, D. Gazit and A. Schwenk, Phys. Rev. D 86, 103511 (2012)

[38] M. Hoferichter, P. Klos, J. Menéndez and A. Schwenk, Phys. Rev. D 94, 063505 (2016)

[39] S. Bacca and S. Pastore, J. Phys. G 41, 123002 (2014)

[40] D. Gazit, S. Quaglioni, and P. Navrátil, Phys. Rev. Lett. 103, 102502 (2009)

[41] A. Ekström et al., Phys. Rev. Lett. 113, 262504 (2014)

[42] J. Engel, F. Šimkovic and P. Vogel, Phys. Rev. C 89, 064308 (2014)

[43] J. Engel, S. Pittel and P. Vogel, Int. J. Mod. Phys. E 1, 1 (1992)

[44] A. L. Fitzpatrick, W. Haxton, E. Katz, N. Lubbers and Y. Xu, JCAP 1302, 004 (2013)

[45] N. Anand, A. L. Fitzpatrick and W. C. Haxton, Phys. Rev. C 89, 065501 (2014)

[46] E. Epelbaum, H. W. Hammer and U.-G. Meißner, Rev. Mod. Phys. 81, 1773 (2009)

[47] R. Machleidt and D. R. Entem, Phys. Rept. 503, 1 (2011)

[48] L. Vietze, P. Klos, J. Menéndez, W. C. Haxton and A. Schwenk, Phys. Rev. D 91, 043520 (2015)

[49] P. Klos, J. Menéndez, D. Gazit and A. Schwenk, Phys. Rev. D 88, 083516 (2013)

[50] D. S. Akerib et al. [LUX Collaboration], Phys. Rev. Lett. 116, 161302 (2016)

[51] L. Baudis, G. Kessler, P. Klos, R. F. Lang, J. Menéndez, S. Reichard and A. Schwenk, Phys.

Rev. D 88, 115014 (2013)

[52] C. McCabe, JCAP 1605, 033 (2016) 